\title{
Deficiency in Homogentisic Acid Oxidase Activity Associated with the Brown Phenotype of Dictyostelium discoideum
}

\author{
By JAMES H. MORRISSEY $\dagger$ \\ Biology Department, B-O22, University of California, San Diego La Jolla, \\ California 92093, U.S.A.
}

(Received 9 July 1982; revised 13 September 1982)

\begin{abstract}
Mutations at the bwnA locus of Dictyostelium discoideum were found to result in drastically reduced levels of activity of the enzyme homogentisic acid oxidase. It is proposed that the brown phenotype associated with these mutations results from the accumulation of an intermediate in the tyrosine degradative pathway, homogentisic acid, and its subsequent spontaneous oxidation. This knowledge is used to devise improved screening procedures potentially useful in the parasexual genetics system of this organism.
\end{abstract}

\section{INTRODUCTION}

The mutation bwnAl of Dictyostelium discoideum, first reported by Sussman \& Sussman (1963), causes the cells which possess it to accumulate a water-soluble pigment over a period of several days that eventually stains both the fruiting bodies and the surrounding agar dark brown. This mutation was found to be useful as a marker for linkage group IV (Katz \& Sussman, 1972) and has since been incorporated into many, if not most, of the tester strains developed for linkage analyses using the parasexual genetics system (for a recent review, see Newell, 1978). Although $b w n A l$ is widely used as a marker, the nature of the brown pigment and the enzymic lesion associated with the mutation have not previously been elucidated.

The brown phenotype has some similarities to alkaptonuria, a human disease identified over seventy years ago by Garrod (see Harris, 1963). Persons suffering from this disease pass urine which turns dark brown when made alkaline. The defect was shown to be due to the lack of homogentisic acid oxidase activity, an enzyme that forms part of the tyrosine and phenylalanine degradative pathway. In its absence homogentisic acid accumulates in the urine and at basic $\mathrm{pH}$ and in the presence of oxygen spontaneously oxidizes and polymerizes to form a melanin-like pigment.

In this report, the $b w n$ mutations are shown to be associated with the loss of homogentisic acid oxidase activity and the concomitant accumulation of homogentisic acid.

\section{METHODS}

Strains and culture conditions. The strains used here all ultimately derive from the wild isolate NC4. Since many have complex genotypes not relevant to this communication, I have chosen to give only their genotypes at two loci: bwnA (which determines pigment production), and sup $A$ (which suppresses pigment production in $b w n A$ strains); full genotypic information may be obtained in the appropriate references. The $b w n^{+} s u p^{+}$strains used in this study are: AX3 (Loomis, 1971); HL33, HL51 and HL100 (Morrissey et al., 1980); HL203 (Morrissey \& Loomis, 1981). The $b w n A l$ sup $^{+}$strains are: X2 (Williams et al., 1974), X9 (Kessin et al., 1974), X36 (Williams \& Newell, 1976), XP95 and XP99 (Ratner \& Newell, 1978). The bwnAl supAl strains are: NP153 (Williams \& Newell, 1976) and HU60 (Welker \& Williams, 1982). Strain HL50 is a spontaneous methanol-resistant derivative of HL100; its genotype is $b w n^{+} a c r A 500 b s g B 500$. HL81 was a gift of S. Wheeler and was obtained following $N$-methyl- $N^{\prime}$-nitro$N$-nitrosoguanidine mutagenesis of AX3. Its genotype is bwn-500 axeAl axeB1. It is phenotypically brown but

$\dagger$ Present address: Department of Biochemistry, University of Oxford, South Parks Road, Oxford OXI 3QU, U.K. 
complementation with bwnAl has not been tested. Strain HL90 was a gift of J. A. Schmidt and was isolated following similar mutagenesis of HL100: it carries $b w n A 50 I$ and $b s g B 500$. The mutation was found to be allelic to bwnAl (unpublished results).

The axenic strains AX3, HL81 and X2 were grown singly in broth medium (Loomis, 1971), and the rest of the strains were grown in association with Klebsiella aerogenes on SM agar (Sussman, 1966).

Brown pigment production. Screening for brown pigment production was performed by shaking amoebae suspended at $1 \times 10^{7}$ cells ml $^{-1}$ in unbuffered Bonner's saline (Bonner, 1947) plus $400 \mu \mathrm{g}$ dihydrostreptomycin sulphate $\mathrm{ml}^{-1}$. When present, amino acids were dissolved by boiling, after which the solution was cooled and adjusted to $\mathrm{pH} 6.2$ by the addition of $\mathrm{HCl}$ or $\mathrm{NaOH}$. Routine screening was usually performed in Amino Acid Saline (AAS, composed of $50 \mathrm{~mm}$-phenylanaline, $5 \mathrm{~mm}$-tyrosine in Bonner's saline plus dihydrostreptomycin sulphate, final pH 6.2) in microtitre trays containing 50 to $100 \mu$ l solution per well. To screen large numbers of colonies it was usually sufficient to scrape cells from the growing edge with a sterile toothpick and suspend them in AAS in a well thus ensuring that the cell concentration was greater than or equal to the minimum cell density $(1 \times$ $10^{7} \mathrm{ml}^{-1}$ ) required for the colour to appear.

Chromatography. Samples were prepared for TLC by shaking cells at $1.0 \times 10^{7} \mathrm{ml}^{-1}$ for $48 \mathrm{~h}$ in 15 mM-sodium phosphate buffer, $\mathrm{pH} 6 \cdot 2$, containing $7 \mathrm{~mm}$-tyrosine. Cells were removed by centrifugation and the supernatant acidified to $\mathrm{pH} 1$ with concentrated $\mathrm{HCl}$. NaCl was added to $22 \%$ and the solution extracted with two volumes of ethyl acetate. The organic layer was removed and concentrated by evaporation at room temperature to one-tenth volume; $10 \mu \mathrm{l}$ of this extract was routinely used per chromatogram.

TLC was carried out according to Kelly (1977), except that silica gel-impregnated glass fibre sheets (ITLC-SG, from Gelman) were used. Detection was accomplished by spraying the chromatogram with $1 \mathrm{~N}$ Folin-Ciocalteau phenol reagent followed by exposure to ammonia fumes.

Enzyme assay. Homogentisic acid oxidase [homogentisate :oxygen 1,2 oxidoreductase (decyclizing), EC 1.13.11.5] activity was measured using the spectrophotometric assay of Takemori et al. (1968). Crude lysates were

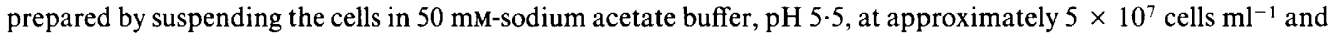
sonicating for $30 \mathrm{~s}$ on ice. The reaction mixture contained $0.1 \mathrm{ml}$ lysate per $\mathrm{ml}$, and the final concentrations of the other constituents were: $167 \mu \mathrm{M}$-homogentisic acid, $667 \mu \mathrm{M}$-ferrous sulphate, and $50 \mathrm{~mm}$-sodium acetate, pH 5.5. Protein concentrations in the lysates were determined by the method of Bradford (1976) using a commercially available preparation (Bio-Rad). A unit of enzyme activity is an amount of enzyme that will produce $1 \mu \mathrm{mol}$ product per minute, and the specific activity is expressed as units per $\mathrm{mg}$ total protein.

\section{RESULTS}

\section{Effect of aromatic amino acids}

To test the hypothesis that the brown phenotype results from an alkaptonuria-like state, the effects of aromatic amino acids on the production of brown pigment were investigated. It had been demonstrated previously that amoebae shaken in Bonner's saline (an unbuffered salt solution) elaborate the brown pigment, which becomes visible by $36 \mathrm{~h}$ (Morrissey et al., 1980). This was repeated using strains X36 (bwnAl) and HL203 $\left(b w n^{+}\right)$, with the addition of varying concentrations of the aromatic amino acids phenylalanine, tyrosine and tryptophan. It was found that adding tyrosine at $5 \mathrm{~mm}$ or phenylalanine at $50 \mathrm{~mm}$ caused the reddish-brown pigment to accumulate within $16 \mathrm{~h}$ and to be much more intense than when X36 cells were shaken in Bonner's saline alone. Tryptophan at concentrations up to $50 \mathrm{~mm}$ was found not to accelerate the appearance or increase the darkness of the pigment produced by X36. HL203 cells did not accumulate the brown pigment in the presence or the absence of any of the amino acids tested.

The pigment was found to be darkest when cells of $b w n$ strains were shaken overnight in a mixture of $50 \mathrm{~mm}$-phenylalanine and $5 \mathrm{~mm}$-tyrosine in Bonner's saline plus dihydrostreptomycin sulphate and this was used as the basis for subsequent screenings.

Homogentisic acid decomposes to give the brown polymer only in basic solution. Accordingly, the $\mathrm{pH}$ of cell suspensions after shaking overnight in AAS (initially at $\mathrm{pH} 6 \cdot 2$ ) was measured and in all cases was found to be greater than 8 . When $10 \mathrm{~mm}$-sodium phosphate buffer (pH 6.2) was substituted for Bonner's saline in AAS, no pigment was found after shaking cells of bwn strains in it overnight (by which time the $\mathrm{pH}$ was still less than 7). However, as might be predicted, making the solution alkaline by adding a few drops of $1 \mathrm{~m}-\mathrm{NaOH}$ caused the brown pigment to appear within a few minutes. 


\title{
Table 1. Effect of amino acids on pigment formation in haploid and diploid strains
}

\begin{abstract}
Cells were shaken at $1.0 \times 10^{7} \mathrm{ml}^{-1}$ with amino acids (in AAS, which contains phenylalanine and tyrosine) or without amino acids (in Bonner's saline containing $400 \mu \mathrm{g}$ dihydrostreptomycin sulphate $\mathrm{ml}^{-1}$ ). Pigment was scored visually after $36 \mathrm{~h}$. Pigmentation was scored subjectively: - denotes the absence of pigment, + denotes pigment was observed, ++ denotes a much more intense brown colour than + was observed.
\end{abstract}

$\begin{array}{llcc}\text { Strain } & \text { Genotype } & \text { With amino acids } & \text { Without amino acids } \\ \text { HL50 } & b w n^{+} & - & - \\ \text {X9 } & b w n A 1 & ++ & + \\ \text { XP99 } & b w n A 1 & ++ & + \\ \text { HL33/XP95 } & b w n^{+} / b w n A 1 & + & - \\ \text { HL51/XP99 } & b w n^{+} / b w n A 1 & ++ & - \\ 90 \% \text { HL50 }+10 \% \text { X9 } & b w n^{+} ; b w n A 1 & - & \text { ND } \\ & \text { ND, Not determined. }\end{array}$

\section{Screening of haploids and diploids}

In addition to speeding up the screening of $b w n$ haploids, shaking cells in AAS can be used to discriminate haploid from diploid cell lines. Table 1 shows the results of shaking cells of several different strains in suspension with or without added amino acids. As before, $b w n^{+}$haploid strains do not form the pigment under either condition, while $b w n A$ haploids do so under both conditions. Diploids heterozygous for the $b w n A$ mutation, however, were found to accumulate the pigment only when challenged with phenylalanine and tyrosine and not when shaken in Bonner's saline alone (Table 1). Diploids homozygous for $b w n^{+}$or $b w n A$ behave like the corresponding haploids (not shown).

Since diploid strains are known to give rise spontaneously to haploid segregants during growth, it is possible that the brown pigment was produced by a few bwnA haploids in the populations of diploid cells used. To test this, samples of the diploid cells used in the experiment in Table 1 were removed from the shaking cultures and plated at low cell density on nutrient agar with bacteria. Colony counts showed that $b w n A$ haploids made up less than $0.4 \%$ of the cells in both cases. That this incidence is too low to account for the production of the pigment is demonstrated by the result in the last line of Table 1 : when a mixture of $10 \% \mathrm{X} 9(\mathrm{bwnAl})$ cells and $90 \%$ HL50 $\left(b w n^{+}\right)$cells were shaken together in AAS, no brown colour resulted.

\section{Chromatography of homogentisic acid}

To identify the substance responsible for the formation of the brown pigment, cells of various strains were shaken overnight in the presence of $5 \mathrm{~mm}$-tyrosine, after which the medium was collected, concentrated, and analysed by TLC. As may be seen in Fig. 1, the three $b w n$ strains HL81, HL90, and X2 (lanes 4-6) accumulated a phenolic substance which co-chromatographed with the purified homogentisic acid standard (lane 1), while no such substance could be detected in the sample from the $b w n^{+}$strain HL100 (lane 3).

\section{Enzyme activities}

Since no studies of the enzyme homogentisic acid oxidase of this organism have, to my knowledge, yet been reported, a brief investigation of its properties in crude lysates of the $b w n^{+}$ strain $\mathrm{AX} 3$ was undertaken. AX3 was used because it grows axenically, eliminating any possible contribution to enzyme activity from contaminating bacteria. The activity was found to be linearly related to the amount of lysate added, and was linear with respect to time for at least 30 min. The reducing agents ascorbate and glutathione were found to be unnecessary at $\mathrm{pH} 5 \cdot 5$. Activity of the enzyme had a strict requirement for added ferrous sulphate, with an apparent $K_{\mathrm{m}}$ for ferrous ion of $1.5 \times 10^{-4} \mathrm{M}$, while the $K_{\mathrm{m}}$ for homogentisic acid was $7 \times 10^{-5} \mathrm{M}$. Developmental time courses, both on filters and of cells shaken in suspension, showed that the activity of the enzyme remained constant throughout development (data not shown).

The enzyme activities of crude lysates of cells of various $b w n^{+}$and $b w n$ strains starved 


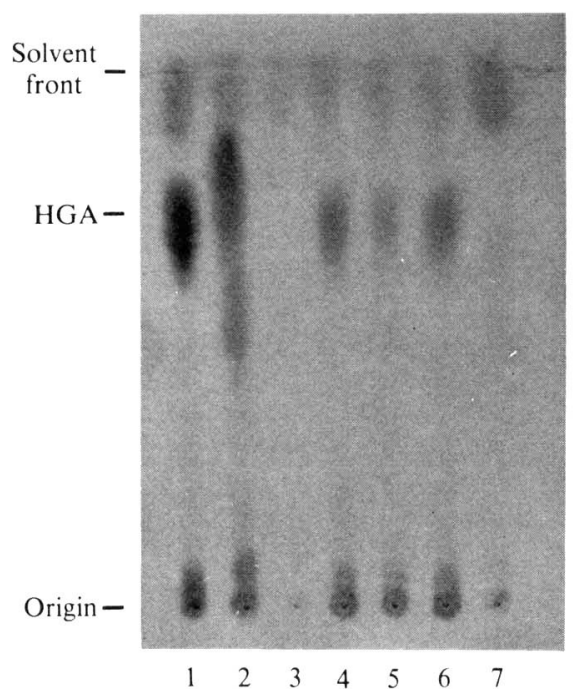

Fig. 1. Thin-layer chromatogram of extracts from $b w n$ and $b w n^{+}$strains developed to reveal phenolic substances. Purified standards are : lane $1,4 \mu \mathrm{g}$ homogentisic acid; lane $2,4 \mu \mathrm{g} p$-hydroxyphenylpyruvic acid. The position to which homogentisic acid chromatographs is marked ' $\mathrm{HGA}$ '. The other lanes contain $10 \mu \mathrm{l}$ samples from the following strains : lane 3, HL100 $\left(b w n^{+}\right)$; lane 4, HL81 $(b w n-500)$; lane 5, HL90 (bwnA50I); lane 6, X2 (bwnAl); lane 7, HU60 (bwnAl supAl).

Table 2. Homogentisic acid oxidase activity in mutant and wild-type strains

Cells were grown in the presence of $K$. aerogenes, then washed free of bacteria and suspended in $15 \mathrm{~mm}-$ sodium phosphate buffer, $\mathrm{pH} 6.2$, at $1.0 \times 10^{7} \mathrm{ml}^{-1}$. After starving in suspension for $16 \mathrm{~h}$ they were collected by centrifugation and assayed as described in Methods. Specific activities are expressed as units per mg total protein, and are the average of three determinations.

$\begin{array}{llc}\text { Strain } & \text { Genotype } & \text { Specific activity } \\ \text { AX3 } & b w n^{+} & 1 \cdot 40 \\ \text { HL100 } & b w n^{+} & 0 \cdot 92 \\ \text { X2 } & b w n A 1 & 0 \cdot 00 \\ \text { HL81 } & b w n-500 & 0 \cdot 14 \\ \text { HL90 } & \text { bwnA501 } & 0 \cdot 14 \\ \text { HU60 } & \text { bwnA1 supAI } & 0.04 \\ \text { NP153 } & \text { bwnAlsupAl } & 0.01\end{array}$

overnight in suspensions are presented in Table 2. As expected, $\mathrm{X} 2$, which carries the $b w n A 1$ mutation, had levels of enzyme activity at or below the limits of detection of the assay, while the $b w n^{+}$strains were found to possess at least 10 times this level of homogentisic acid activity. The two new bwn mutations, $b w n-500$ and $b w n A 501$, resulted in barely detectable levels of this enzyme.

Williams (1978) has reported that cobalt-resistant strains which are genotypically $b w n^{+}$ exhibit a brown phenocopy when grown in the presence of cobaltous chloride. Cobalt at concentrations employed by Williams (1978) $\left(350 \mu \mathrm{g} \mathrm{CoCl}_{2} \cdot 6 \mathrm{H}_{2} \mathrm{O} \mathrm{ml}{ }^{-1}\right)$ completely inhibited homogentisic acid oxidase in vitro (data not shown).

\section{Suppressor of bwnA mutations}

A mutation (supAl), discovered by Williams \& Newell (1976) suppresses the formation of brown pigment in strains carrying $b w n A$. This mutation was shown to be unlinked to $b w n A$ (D) Mosses \& P. C. Newell, personal communication; see also Welker \& Williams, 1982). Figure 1, 
lane 7 , indicates that a strain carrying both $\sup A 1$ and $b w n A l$ does not produce homogentisic acid. The supAI mutation does not exert its effect by restoring homogentisic acid oxidase activity to $b w n A$ strains (Table 2, strains HU60 and NP153). Since there are two enzymic steps between phenylalanine and homogentisic acid, it was considered that the suppressor may cause the loss of either tyrosine transaminase or 4-hydroxyphenylpyruvate oxidase activity. The activities of these enzymes were measured in crude lysates of $b w n A 1$ supAl strains, but were found to be unaltered relative to wild-type (both $b w n^{+}$and $b w n A$ strains; data not shown).

\section{DISCUSSION}

The results are consistent with the idea that the $b w n A$ mutation results in a deficiency of homogentisic acid oxidase activity, which causes the accumulation in the medium of homogentisic acid formed from the breakdown of phenylalanine and tyrosine. Under alkaline conditions, homogentisic acid undergoes oxidation and polymerization to form a characteristic brown, melanin-like pigment. Dictyostelium discoideum cells are known to secrete ammonia in millimolar amounts during development (Schindler \& Sussman, 1977), which creates the alkaline $\mathrm{pH}$ necessary for the formation of pigment from homogentisic acid. This explains why the brown colour does not form in buffered cell suspensions unless the $\mathrm{pH}$ is raised artificially. It also explains why the brown colour from cells grown on nutrient agar plates takes some 7-9 d to appear: presumably it takes this long for sufficient ammonia to be secreted in order to overcome the buffers in the growth medium. I have found that the $\mathrm{pH}$ of the agar of $8 \mathrm{~d}$ old growth plates is usually in excess of 8 (unpublished observations).

The association between $b w n A$ and the loss of homogentisic acid oxidase activity is unlikely to be fortuitous because it exists in several bwnAl strains that have been extensively outcrossed, and more importantly, was demonstrated in two new isolates, HL81 $(b w n-500)$ and HL90 ( $b w n A 501$ ). These are the only new $b w n$ mutations described since the first descriptions of $b w n A l$ in 1963.

Although precocious appearance of the melanin-like pigment may be obtained by exposing fruiting bodies of $b w n$ strains to ammonia fumes (unpublished results), this is not recommended because it kills the cells and stops any further pigment production. Instead the method involving shaking amoebae in a solution containing phenylalanine and tyrosine is preferred. A dual screening technique (with and without added amino acids) has been proposed which is capable of discriminating heterozygous $b w n^{+} \mid b w n A$ diploids from either of the haploid parents. This can be used to determine the ploidy of putative diploids obtained from fusions of $b w n A$ and $b w n^{+}$ haploid strains, and should be particularly useful with non-sporulating mutants such as aggregateless $(a g g)$ or stalky $(s t k)$. This is because in such cases it is not possible to use the characteristic size and shape of the spores (Sussman \& Sussman, 1963) as a measure of ploidy. The alternative, karyotyping (Brody \& Williams, 1974), is rather more laborious.

The suppressor mutation, sup $A$, did not restore homogentisic acid oxidase activity, and further did not result in a deficiency in either of the two preceding enzymes in the phenylalanine degradative pathway. These findings are perhaps not surprising in light of the recent discovery that supAI suppresses cobalt resistance as well as brown pigment formation (K. L. Williams, personal communication), indicating that it may exert a more general effect.

The brown phenocopy found when strains are grown in the presence of cobalt chloride (Williams, 1978) apparently results from the inhibition of homogentisic acid oxidase by cobaltous ion. A plausible explanation of this is that the cobaltous ion may displace the ferrous ion known to be required for activity of this enzyme.

I would like to acknowledge the help and advice of Dr William F. Loomis, in whose laboratory this study was conducted, and I would like to thank J. A. Schmidt, S. Wheeler and K. L. Williams for their generous gifts of the unpublished strains HL90, HL81, and HU60, respectively. I would also like to thank K. L. Williams and P. C. Newell for their critical reading of the manuscript, and S. McRobbie for helpful suggestions. This work was supported by a grant to W. F. L. from the National Science Foundation (PCM 02698). J. H. M. was supported by an institutional postdoctoral training grant from the National Institutes of Health (GM 07199-06). 


\section{REFERENCES}

BONNER, J. T. (1947). Evidence for the formation of cell aggregates by chemotaxis in the development of the slime mold Dictyostelium discoideum. Journal of Experimental Zoology 106, 1-26.

BRADFORD, M. (1976). A rapid and sensitive method for the quantitation of microgram quantities of protein utilizing the principle of protein-dye binding. Analytical Biochemistry 72, 248-254.

BRoDY, T. \& Williams, K. L. (1974). Cytological analysis of the parasexual cycle in Dictyostelium discoideum. Journal of General Microbiology 82, 371383.

HARRIS, H. (1963). Garrod's Inborn Errors of Metabolism. London: Oxford University Press.

Katz, E. R. \& Sussman, M. (1972). Parasexual recombination in Dictyostelium: selection of stable diploid heterozygotes and stable haploid segregants. Proceedings of the National Academy of Sciences of the United States of America 69, 495-498.

Kelly, S. (1977). Biochemical Methods in Medical Genetics. Springfield, Illinois: C. C. Thomas.

Kessin, R. H., Williams, K. L. \& Newell, P. C. (1974). Linkage analysis in Dictyostelium discoideum using temperature-sensitive growth mutants selected with bromodeoxyuridine. Journal of Bacteriology 119, 959-962.

LоOмIS, W. F., JR (1971). Sensitivity of Dictyostelium discoideum to nucleic acid analogues. Experimental Cell Research 64, 484-486.

Morrissey, J. H. \& LoOmis, W. F. (1981). Parasexual genetic analysis of cell proportioning mutants of Dictyostelium discoideum. Genetics 99, 183-196.

Morrissey, J. H., Wheeler, S. \& LoOMIS, W. F. (1980). New loci in Dictyostelium discoideum determining pigment formation and growth on Bacillus subtilis. Genetics 96, 115-123.
Newell, P. C. (1978). Genetics of the cellular slime moulds. Annual Review of Genetics 12, 69-93.

Ratner, D. I. \& Newell, P. C. (1978). Linkage analysis in Dictyostelium discoideum using multiply marked tester strains: establishment of linkage group VII and the reassessment of earlier linkage data. Journal of General Microbiology 109, 225-236.

Schindler, J, \& Sussman, M. (1977). Ammonia determines the choice of morphogenetic pathways in Dictyostelium discoideum. Journal of Molecular Biology 116, 161-169.

SUSSMAN, M. (1966). Biochemical and genetic methods in the study of cellular slime mold development. Methods in Cell Physiology 2, 397-410.

Sussman, R. R. \& Sussman, M. (1963). Ploidal inheritance in the slime mould Dictyostelium discoideum. Journal of General Microbiology 30, 349-355.

Takemori, S., Furuya, E., Mihara, K. \& Katagiri, M. (1968). Bovine liver homogentisicase: apo- and reconstituted holoenzymes. European Journal of Biochemistry 6, 411-418.

Welker, D. L. \& Williams, K. L. (1982). A genetic map of Dictyostelium discoideum based on mitotic recombination. Genetics $102,691-710$.

Williams, K. L. (1978). Characterization of dominant resistance to cobalt chloride in Dictyostelium discoideum and its use in parasexual genetic analysis. Genetics 90, 37-47.

Williams, K. L. \& Newell, P. C. (1976). A genetic study of aggregation in the cellular slime mould Dictyostelium discoideum using complementation analysis. Genetics 82, 287-307.

Williams, K. L., Kessin, R. H. \& Newell, P. C. (1974). Parasexual genetics in Dictyostelium discoideum: mitotic analysis of acriflavin resistance and growth in axenic medium. Journal of General Microbiology 83, 59-69. 